\title{
Tooth erosion and the role of pepsin reflux
}

\author{
Jeanine Fisher ${ }^{1}$, Graham Porter ${ }^{2}$, Stuart Graham², Christos \\ Ziaras $^{2}$, Andrew D Woodcock ${ }^{1}$ and Peter W Dettmar ${ }^{1 *}$ \\ ${ }^{1}$ RD Biomed Limited, Castle Hill Hospital, Cottingham, HU16 5JQ, UK \\ ${ }^{2}$ Graham Porter Dentistry, Castle Green, Cottingham, HU16 5JU, UK
}

\section{Abstract}

Objective: The purpose of the study was to evaluate if there is a link between salivary pepsin levels and tooth erosion. Also, to determine if gastroesophageal reflux disease (GERD) is responsible for much of the tooth erosion seen by dentists.

Background: Pepsin is only produced within the stomach. If found within other parts of the body [for example within saliva or sputum samples], the only mechanism by which that would be possible is via the reflux of gastric contents. One of the causes of dental erosion is thought to be due to direct contact between tooth surfaces and acidic substances and digestive enzymes present in gastric refluxate. GERD is a common condition, with its prevalence seemingly trending higher in recent decades. It is reportedly a known cause of tooth erosions. From the hypothesis, there was an expectation to see patients with dental erosions to have pepsin detected [and perhaps at high levels] and to see patients without dental erosions to have no or low levels of pepsin.

Method: Three saliva samples were collected [on waking and 2 post-prandial] from 50 anonymous participating patients ( 26 females, 24 males) from a single dental practice. Extra information was collected related to lifestyle, Reflux Symptom Index (RSI - reflux questionnaire) and tooth erosions. These samples were analyzed for the stomach enzyme pepsin using the validated medical device Peptest.

Results: There was no correlation between positive pepsin levels and the presence of tooth erosion during this study. There was a statistical difference between the on waking $\mathrm{pH}$ vs. positive pepsin levels and post prandial $\mathrm{pH}$ vs. positive pepsin levels. The average $\mathrm{pH}$ was lower for on waking and post-prandial samples with positive pepsin, suggesting that the saliva was acidic and gastric reflux had occurred. Conversely, the average $\mathrm{pH}$ was higher for on waking and postprandial samples with negative pepsin. There was no statistical difference between $\mathrm{pH}$ vs. tooth erosion in the on waking and post- prandial.

Conclusion: Patients identified as having tooth erosion did not have higher levels of pepsin detected, suggesting that pepsin was not associated with dental erosion in these patients.

\section{More Information}

*Address for Correspondence: Peter W Dettmar, RD Biomed Limited, Daisy Building, Castle Hill Hospital, Castle Road, Cottingham, East Yorkshire, HU16 5JQ, UK, Tel; +44 [0] 1482 461877; Email: peter.dettmar@technostics.com

\section{Submitted: 15 April 2020 \\ Approved: 22 April 2020 \\ Published: 24 April 2020}

How to cite this article: Fisher J, Porter G, Graham S, Ziaras C, Woodcock AD, et al. Tooth erosion and the role of pepsin reflux. J Clin Adv Dent. 2020; 4: 009-014.

DOI: 10.29328/journal.jcad.1001016

Copyright: ( 2020 Fisher J, et al. This is an open access article distributed under the Creative Commons Attribution License, which permits unrestricted use, distribution, and reproduction in any medium, provided the original work is properly cited.

Keywords: Pepsin; Dental; GERD; Peptest; Reflux; Tooth erosion

Check for updates

OPEN ACCESS

\section{Introduction}

Dental erosion is caused by sustained direct contact between tooth surfaces and acidic substances, this is a common condition, with its prevalence seemingly trending higher in recent decades [1]. Dental erosion is defined as the irreversible loss of tooth structure due to the chemical dissolution via the acidic substances.

Dental erosion is a multifactorial condition. The consideration of chemical, biological and behavioral factors is fundamental for its prevention and therapy. Among the biological factors, saliva is one of the most important parameters in the protection against erosive damage [2].
Pepsin is an enzyme, discovered in the eighteenth century, and the first discovered enzyme as well as the second enzyme to be crystallized [3]. Specifically, the proteolytic enzyme pepsin is found predominately within the stomach, expressed initially as its precursor substance pepsinogen, known as a zymogen, found within the lining of stomach cells. Chief cells within the stomach secrete pepsinogen which is then activated via hydrochloric acid (HCI) (secreted via the parietal cells within the stomach lining), the HCI within the gastric juice converting the inactive form pepsinogen into the active form that is the enzyme pepsin (Figure 1). Pepsin has the capability to degrade food and proteins into peptides, ultimately producing digestion. Being a digestive protease, 


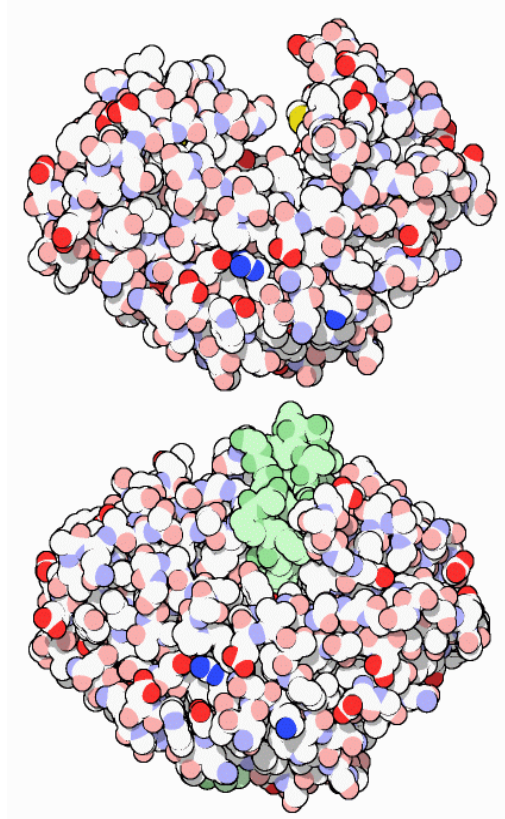

Figure 1: Pepsin - aspartic proteinase and major component of gastric refluxate. Family of pepsin isoenzymes active up to $\mathrm{pH} 6.5$ and not denatured until $\mathrm{pH} 7.8$.

pepsin is among three substances that aid in the digestion process however pepsin is the main component amongst all stomach juices with the digestion process unable to complete without it being present.

If pepsin is detected in saliva it suggests it has been refluxed into the throat/mouth indicating reflux disease which consequently causes dental erosion. Gastric acid has been demonstrated to displace saliva from tooth surfaces $[2,4]$. Increasing incidence and high prevalence of dental erosions are associated with tooth sensitivity observed in many countries worldwide, within both children and adults [5]. Within the present pilot study, we predicted to see patients with dental erosions to have pepsin detected and patients without dental erosions to have no or low levels of pepsin.

\section{Methods and Material}

\section{Recruitment}

Fifty consecutive patients attending Graham Porter Dentistry (Cottingham, UK) were invited to participate in the current study. East Midlands - Nottingham 1 Research Ethics Committee approved the study (REC reference 16/EM/0409). All study patients were asked to provide three unstimulated saliva samples by spitting into a collection tube providing a $1 \mathrm{ml}$ saliva sample, one upon waking before eating and cleaning teeth and two post-prandial samples provided 60 minutes after eating lunch and dinner. All samples were anonymised prior to pepsin analysis conducted in the RD Biomed Limited central laboratory at Castle Hill Hospital, Cottingham. RD Biomed Limited received no identifiable information linking samples to study patients. This was achieved via the patient recruitment taking place within the dental practice. The staff at the dental practice were given training regarding pepsin/
Peptest in the event that patients had any questions which had not been addressed within the patient information sheet.

RD Biomed Limited provided the dental practice with an excel spreadsheet containing a list of codes, with each code representing a study patient. The dental practice assigned a code to each patient, noting the patients name next to the allocated code and whether they had tooth erosions or not. The spreadsheet was password protected with no access given to RD Biomed Limited.

Study patients received a consent form to sign. Patients were provided with the saliva collection tubes and instructions at the dental practice and asked to produce saliva samples at home and return the samples [using the provided free-post envelope] to the laboratory for analysis. All patients completed a reflux questionnaire (Reflux Symptom Index, RSI) [6] to establish if they have reflux symptoms. Saliva samples were analysed for pepsin using Peptest.

At each visit patients attending the dental practice were routinely examined for the presence of dental erosions and re-examined at each subsequent visit. In the current study for each patient recruited into the study the dentist was requested to record if dental erosion was present or not present.

\section{Pepsin analysis}

The three collection tubes containing the saliva samples were centrifuged for 5 minutes at $4000 \mathrm{rpm}$ until a clear supernatant layer was visible. From the surface layer $80 \mu \mathrm{L}$ was drawn up using an automatic pipette. The $80 \mu \mathrm{L}$ sample was transferred to a microtube containing $240 \mu \mathrm{L}$ of Migration Buffer. The $320 \mu \mathrm{L}$ mixture was vortexed for 10 seconds. Using a pipette $80 \mu \mathrm{L}$ of the mixture was transferred to the circular well of a Lateral Flow Device (LFD) containing two unique human monoclonal antibodies; one to detect and one to capture any pepsin present in the clinical sample (Peptest, RD Biomed Limited, UK) (Figure 2). A 'control' line was produced within the window of the LFD if the test was successful and a test line was generated if the sample contained pepsin. The LFD was transferred to a Peptest Cube reader which measured the intensity of the test line and gave a concentration of pepsin within the saliva sample in $\mathrm{ng} / \mathrm{mL}$.

\section{Statistical analysis}

Unpaired t tests were performed where possible using the statistical package GraphPad Prism 8.4.0 (GraphPad Software, San Diego, CA 92018, USA). $p$ values $<0.05$ were considered statistically significant.

\section{Resullts}

A total of 50 patients attending a single dental practice were recruited and their demographic information anonymised prior to entry into the study. The gender split was 26 females and 24 males with an average age of 60 and an age range between 25 and 85 . This was a wide age range but 

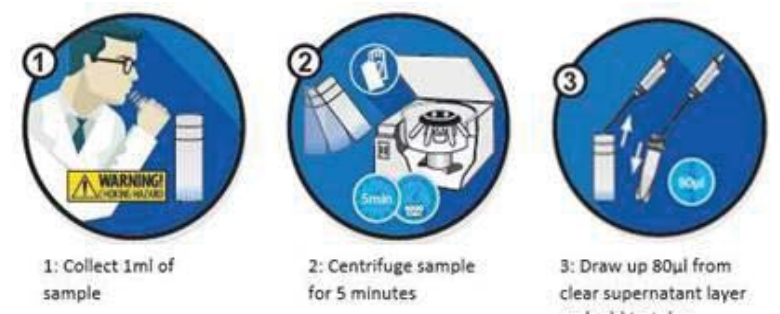

3: Draw up 80ul from clear supernatant layer
and add to tube containing $240 \mu$ migration buffer

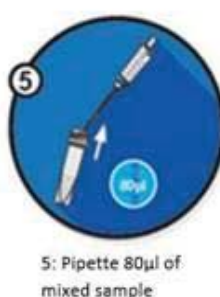

mixed sample

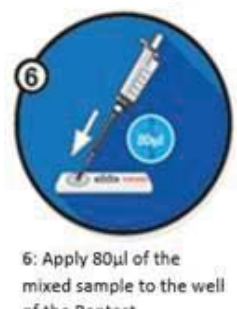

of the Peptest

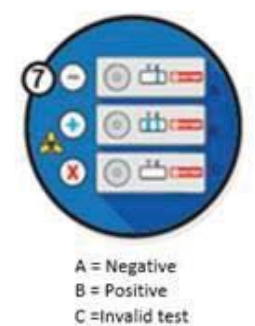

Figure 2: Schematic process for the collection and analysis of saliva samples for the use of Peptest.

even patients at the top end of the age range had no difficulty in providing a one $\mathrm{ml}$ saliva sample. Each of the 50 patients recruited were invited to produce three saliva samples, one in the morning upon waking before breakfast and cleaning teeth and two post-prandial samples 60 minutes after two separate meals usually lunch and dinner. From the 50 recruited patients a total of 150 samples were analyzed for salivary pepsin using Peptest. Figure 3 shows the mean pepsin levels in the female and male patients entered into the study which was significantly higher $(p<0.0367)$ in the female patients. $\mathrm{pH}$ plays a key role in dental erosions and it was important to test the $\mathrm{pH}$ of all the saliva samples provided during the study. $\mathrm{pH}$ was compared in the on waking and post-prandial saliva samples with the presence of positive and negative pepsin in non-erosive and erosive tooth decay.

Figure 4 illustrates the number of patients who provided a saliva sample on waking which were found to be either pepsin positive or pepsin negative in patients with dental erosions compared to patients with no dental erosions.

The concentration of pepsin in the patients with dental erosions ( $n=9$ ) was $200.9 \mathrm{ng} / \mathrm{ml}$ and in the patients, who had no detected dental erosions $(n=4)$ the pepsin concentration was $311.8 \mathrm{ng} / \mathrm{ml}$, this difference was not significant. The surprising observation was the fact there were more patients with no salivary pepsin presenting with dental erosion compared to patients with dental erosions and positive pepsin.

Figure 5 illustrates the number of patients with dental erosions and positive pepsin and negative pepsin in postprandial samples compared to patients presenting with no dental erosions.

The pepsin mean concentration in the group with dental erosions was $145.3 \mathrm{ng} / \mathrm{ml}$ and the group with no dental erosions the pepsin concentration was $251.6 \mathrm{ng} / \mathrm{ml}(p<0.05)$.
In contrast to the on waking salivary pepsin samples shown in figure 4 in the post-prandial saliva samples the number of patients with dental erosions who had either positive pepsin $(n=21)$ or negative pepsin $(n=18)$ was similar.

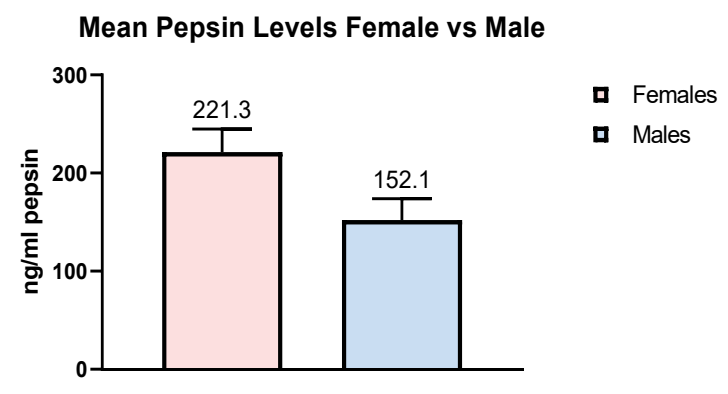

Figure 3: Comparison of pepsin levels in female and male patients.

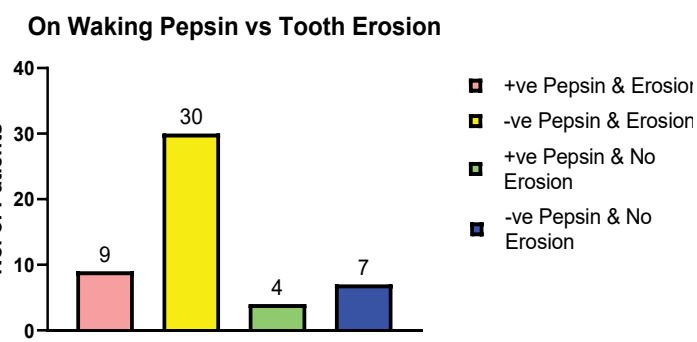

Figure 4: The number of erosive and non-erosive dental patients providing pepsin positive or pepsin negative saliva samples on waking.

Post-Prandial Pepsin vs Tooth Erosion

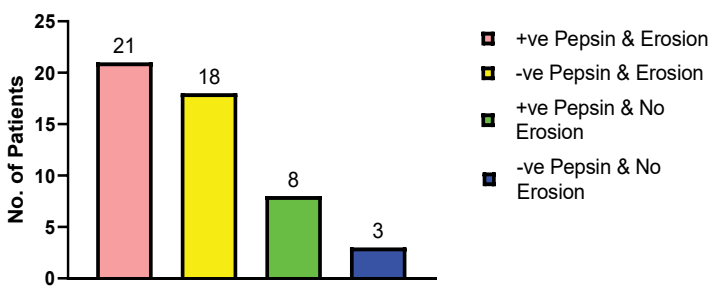

Figure 5: The number of erosive and non-erosive dental patients providing pepsin positive or pepsin negative post-prandial saliva samples. 
Salivary pH plays a key role in being a causal factor in dental erosions. $\mathrm{pH}$ was compared in the on waking and in post-prandial saliva samples with the presence of positive and negative pepsin and in patients with erosive and non-erosive tooth decay.

Figure 6 illustrates the difference in the on waking saliva samples between patients with positive pepsin $(n=13)$ and those with no pepsin $(n=36)$. The mean $\mathrm{pH}$ of the saliva in the pepsin positive patients was $\mathrm{pH} 5.2$ and in the patients with negative pepsin the mean salivary $\mathrm{pH}$ was 6.6. The pepsin positive patients having a lower $\mathrm{pH}$ than the pepsin negative patients with a statistical difference of $p<0.001$.

There was no significant difference in salivary $\mathrm{pH}$ in the on waking saliva samples from patients presenting with dental erosions $(n=38)$ to those with no dental erosions $(n=11)$, (Figure 7). The mean $\mathrm{pH}$ of the saliva in the group with dental erosions was $\mathrm{pH} 6.2$ and the mean $\mathrm{pH}$ of the saliva in the patients with no dental erosions was $\mathrm{pH}$ 6.1.

In the patients with positive salivary pepsin $(n=30)$ in their post-prandial saliva sample there was a significantly ( $p$ $<0.05$ ) lower mean, pH 5.9, compared to those patients with negative salivary pepsin $(n=-20)$ who had a mean $\mathrm{pH}$ of 6.7. The pepsin positive patients having a lower salivary $\mathrm{pH}$ than those patients with negative pepsin, a result similar to that observed in the saliva samples provided on waking (Figure 8).

In the post-prandial saliva samples, there was no significant difference in the $\mathrm{pH}$ between patients presenting with dental erosions $(n=39)$, $\mathrm{pH} 6.2$, and in patients presenting with no dental erosions $(n=11)$, pH 6.2 (Figure 9).

On the combined patient on waking and post-prandial pepsin positive $(n=43)$ and pepsin negative $(n=56]$ salivary $\mathrm{pH}$ samples there was a significant difference $(p<0.0001)$ between the mean $\mathrm{pH}$ of the group samples. The pepsin positive saliva samples had a mean $\mathrm{pH}$ of 5.7 and the pepsin negative samples had a mean $\mathrm{pH}$ of 6.6.

There was no significant difference observed between the salivary $\mathrm{pH}$ samples of patients presenting with dental erosions $(n=77), \mathrm{pH} 6.2$ and patients with no dental erosions $(n=22), \mathrm{pH} 6.2$.

The mean waking salivary pepsin concentrations was $235.0 \mathrm{ng} / \mathrm{ml}$ ( $n=13$ samples] compared to the mean postprandial salivary pepsin concentration which was $175.3 \mathrm{ng} /$ $\mathrm{ml}(n=46$ samples]. The difference in pepsin concentration was not significant. The data is illustrated in figure 10 .

The mean pepsin concentration in patients presenting with no dental erosions was $265.7 \mathrm{ng} / \mathrm{ml}(n=17$ samples] and in patients with dental erosion the mean pepsin concentration was lower at $157.2 \mathrm{ng} / \mathrm{ml}(n=42$ samples]. There was a significant difference at $p<0.05$. The data is illustrated in figure 11.

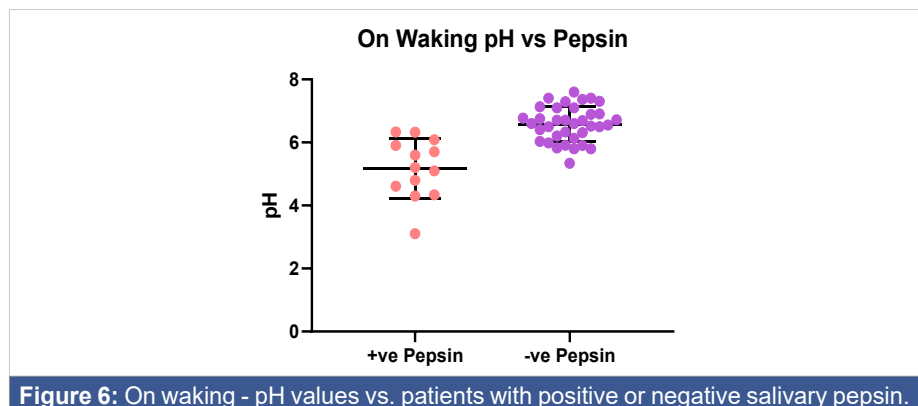

Figure 6: On waking - pH values vs. patients with positive or negative salivary pepsin.

On Waking pH vs Erosion

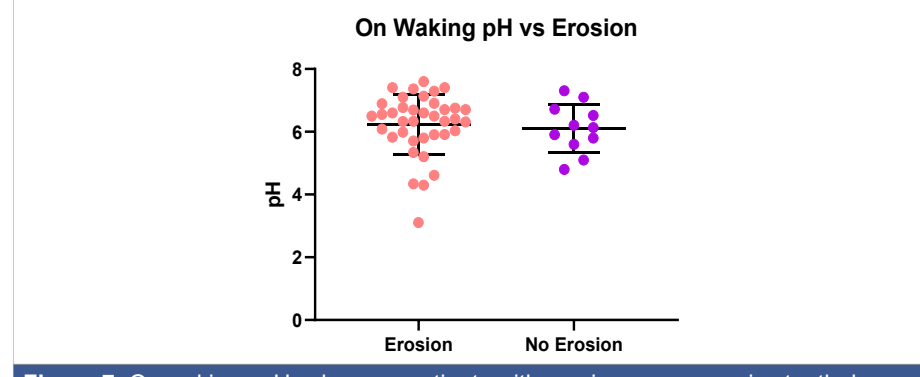

Figure 7: On waking - pH values vs. patients with erosive or non-erosive tooth decay.

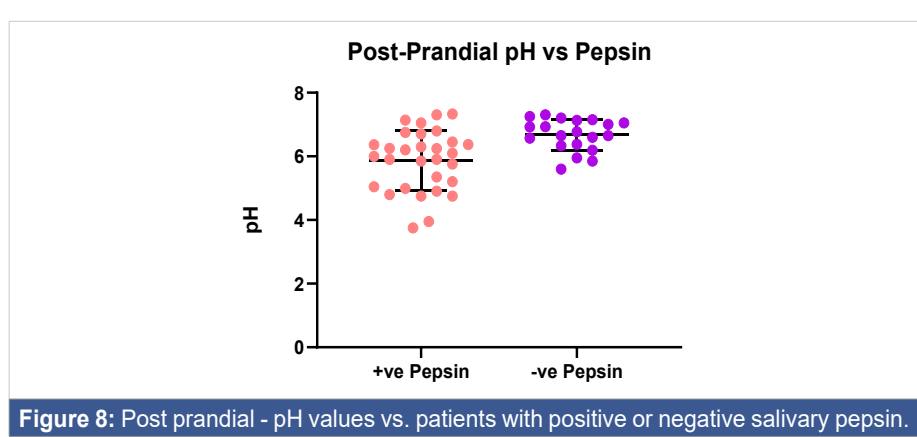

Post-Prandial pH vs Erosion

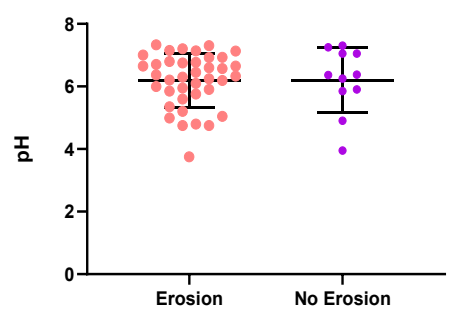

Figure 9: Post prandial - pH values vs. patients with erosive or non-erosive tooth decay.

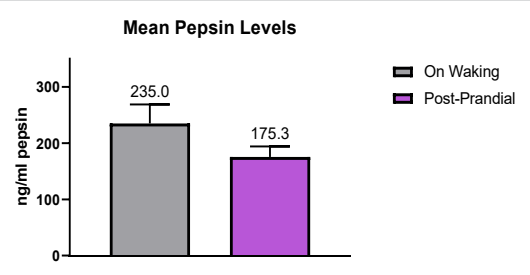

Figure 10: Mean pepsin levels in saliva samples provided on waking and post-prandial.

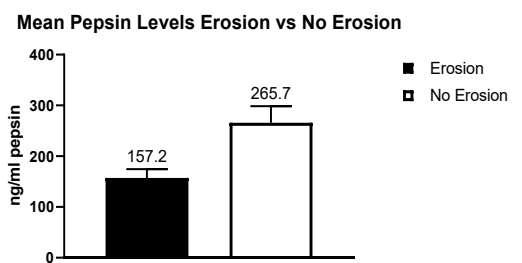

Figure 11: Mean pepsin levels in patients with dental erosions compared to patients with no dental erosions. 


\section{Discussion}

The classification of dental erosions has been well documented, and nomenclatures and classifications described in early reports published, for example, in 1892 by Darby [7], in 1907 by Miller [8] and in 1923 by Pickerill [9]. Classification of tooth erosion [10] is termed either extrinsic, intrinsic or idiopathic. Extrinsic erosions are the result of exogenous acids for example by airborne acidic contaminates. Intrinsic erosions which are the result of endogenous acid, which is highly relevant to the current study, resulting in gastric acid contacting the teeth during, for example, regurgitation of reflux and vomiting. Finally, idiopathic erosions are the result of acids of unknown origin.

Saliva has been described as the most important biological factor related to the development of dental erosions [2]. Dental erosions in the general population are estimated to be around $42 \%$ [11], this percentage is similar to that reported for GERD in Western countries such as in Europe and the USA [12]. Although not every person with GERD will necessarily present with dental erosions. A recent review found a median prevalence of $24 \%$ for tooth erosion in patients with GERD and a median prevalence of $32.5 \%$ for GERD in adult patients who had dental erosions [13]. The common factor in reflux disease and dental erosion is gastric refluxate which contains erosive agents such as acid and pepsin which have been identified as causal in GERD and acid has been associated strongly with dental erosions. Various case studies have concluded that patients diagnosed with GERD are at a higher risk of developing dental erosions $[11,14,15]$. Both endogenous [intrinsic] and exogenous [extrinsic] sources of acids are responsible for the increasing incidence and high prevalence of tooth erosion and associated tooth sensitivity [5].

However, unlike acid which is known to play a major role in causing dental erosion there are very few studies which reference pepsin being associated with dental erosions. Pepsin is a highly aggressive digestive enzyme and present in gastric refluxate causing damage to the upper gastrointestinal epithelium, the airways and lungs [16]. A previous dental study showed that pepsin was capable of completely degrading the demineralised organic matrix of dentine when immersed in pepsin for more than 3 days which is a rather artificial situation $[17,18]$. There has been one previous study investigating the effect of a pepsin-hydrochloric acid solution on erosive dental substance losses and whether pepsin has any influence on the efficacy of fluorides. The outcome was that pepsin had no influence on dental tissue loss but did alter the efficacy of fluoridation measures [19].

GERD is an important cause of dental erosions and defined as the passage of gastric refluxate containing acid and pepsin into the esophagus. In healthy people the gastric refluxate is returned to the stomach by peristalsis stimulated by swallowing. In contrast patients with GERD have delayed acid clearance and patients with dental erosions are less able to clear the acid and pepsin from the esophagus [20]. It is well documented that acid at low pH is strongly associated with demineralisation of the tooth enamel which in turn leads to loss of tooth structure and tooth erosion. The present study set out to establish the role of salivary pepsin in tooth erosion with pepsin determined by a non-invasive diagnostic device (Peptest, RD Biomed Limited, UK) that enabled the rapid determination of pepsin in the collected saliva samples [2125]. The pepsin diagnostic test demonstrated sensitivity and specificity in clinical validation studies [26,27]. This study provided evidence that pepsin was not readily associated with dental erosions with higher pepsin levels found in the salivary pepsin from patients without dental erosions. Pepsin is a larger molecule [16] than acid/HCL [28] and too large to penetrate the tooth enamel and cause a breakdown in dentine, whereas acid being smaller easily penetrates resulting in demineralisation and tooth erosion/decay. There is still the intriguing question of why the patients who had no dental erosions presented with higher levels of pepsin whereas the patients with dental erosions had negative or low pepsin levels present. On first seeing this result we speculated that $\mathrm{pH}$ was involved. However, our data did not support the involvement of $\mathrm{pH}$ as shown in our data analysis. This remains an unanswered question and will be the subject of further investigation.

The main study limitations are the relatively low patient numbers, the study would have benefited from the recruitment of more dental practices. The age range used in this study was wide between 25 and 85 years of age but using older patients did not affect the provision of small one ml samples of saliva. However, future studies might benefit from recruiting and evaluating patients in a narrower age range for example 30 to 70 years of age. The study would have also benefited from determining the level of tooth erosion in each patient not just present or not present.

\section{Conclusion}

Saliva samples provided on waking generally had higher pepsin concentrations than those saliva samples provided post-prandial. Patients with dental erosions had significantly lower salivary pepsin concentrations in both on waking and post-prandial samples compared to those patients presenting with no dental erosions.

We hypothesised that there would be a strong link between salivary pepsin levels and tooth erosion and the aim of this study was to demonstrate this. However, there was no correlation between the presence of dental erosions and the presence of pepsin.

\section{Acknowledgment}

The authors would like to thank Sue Haseltine for recruiting patients into the study and ensuring that they were fully instructed on all study procedures. 


\section{References}

1. Enam $F$, Mursalat $M$, Guha $U$, Aich $N$, Anik M, et al. Characterizing Dental Erosion Potential of Beverages and Bottled Drinking Water in Bangladesh. 4th International Conference on Chemical Engineering (ICChE); Dhaka, Bangladesh. 2014.

2. Buzalaf MAR, Hannas AR, Kato MT. Saliva and dental erosion. J Appl Oral Sci. 2012; 20: 493-502.

PubMed: https://www.ncbi.nlm.nih.gov/pubmed/23138733

3. Fruton Joseph S. A History Of Pepsin And Related Enzymes. Q Rev Biol. 2002; 77: 127-147.

PubMed: https://www.ncbi.nlm.nih.gov/pubmed/12089768

4. Wilder-Smith $\mathrm{CH}$, Materna $\mathrm{A}$, Martig L, Lussi A. Longitudinal study of gastroesophageal reflux and erosive tooth wear. BMC Gastroenterol I. 2007; 17:113.

PubMed: https://www.ncbi.nlm.nih.gov/pubmed/29070010

5. Ranjitkar S, Kaidonis JA, Smales RJ. Gastroesophageal reflux disease and tooth erosion. International journal of dentistry. 2012; 479--850. PubMed: https://www.ncbi.nlm.nih.gov/pmc/articles/PMC3238367/

6. Belafsky PC, Postma GN, Koufman JA. Validity and reliability of the reflux symptom index (RSI), Journal of Voice: Official Journal of the Voice Foundation. 2002; 274-277.

PubMed: https://www.ncbi.nlm.nih.gv/pubmed/12150380

7. Darby ET. Dental Erosion and the Gouty Diathesis. Am J Dent Sci. 1892; 26: 352-355.

PubMed: https://www.ncbi.nlm.nih.gov/pubmed/3074960 7

8. Miller W. Experiments and observations on the wasting of tooth tissue visually variously designated as erosion, abrasion, chemical abrasion, denudation. The Dental Cosmos. 1907: 19: 731-734.

9. Pickerill $\mathrm{H}$. The prevention of dental caries and oral sepsis. London: Bailliere, Tindall \& Cox. 1923: 140.

10. Imfeld T. Dental erosion. Definition, classification and links. Eur J Oral Sci. 1996; 102: 151-155.

PubMed: https://www.ncbi.nlm.nih.gov/pubmed/8804882

11. Cengiz S, Cengiz MI, Saraç YS. Dental erosion caused by gastroesophageal reflux disease: a case report. Cases J. 2009; 2: 8018 . PubMed: https://www.ncbi.nlm.nih.gov/pmc/articles/PMC2740145/

12. El-Serag HB, Sweet S, Winchester CC. Update on the epidemiology of gastro-oesophageal reflux disease: a systematic review. Gut. 2014; 63: 871-880.

PubMed: https://www.ncbi.nlm.nih.gov/pubmed/23853213

13. Pace F, Pallotta S, Tonini M, Vakil N, Bianchi Porro G. Systematic review: gastro-oesophageal reflux disease and dental lesions. Aliment Pharmacol Ther. 2008; 27: 1179-1180.

PubMed: https://www.ncbi.nlm.nih.gov/pubmed/18373634

14. Heidelbaugh JJ, Gill AS, Van Harrison R, Nostrant TT. Atypical presentations of gastroesophageal reflux disease. Am Fam Physician. 2008; 78: 483-488.

PubMed: https://www.ncbi.nlm.nih.gov/pubmed/18756656

15. Bruno V, Amato M, Catapano S, lovino P. Dental erosion in patients seeking treatment for gastrointestinal complaints: a case series. 2015. PubMed: https://www.ncbi.nlm.nih.gov/pubmed/26519024

16. Bardhan KD, Strugala V, Dettmar PW. Reflux Revisited: Advancing the Role of Pepsin. Int J Otolaryngol. 2012; 1-13.

PubMed: https://www.ncbi.nlm.nih.gov/pubmed/22242022

17. Tonami K, Ericson D. Protein profile of pepsin-digested carious and sound human dentine. Acta Odontol Scand. 2005 Feb;63 (1):17-20. PubMed: https://www.ncbi.nlm.nih.gov/pubmed/16095057

18. Kleter GA, Damen JJ, Buijs MJ, Ten Cate JM. The Maillard reaction in demineralized dentin in vitro. Eur J Oral Sci. 1997; 105: 278-284. PubMed: https://www.ncbi.nlm.nih.gov/pubmed/9249196

19. Schlueter N, Ganss C, Hardt M, Schegietz D, Klimek J. Effect of pepsin on erosive tissue loss and the efficacy of fluoridation measures in dentine in vitro. Acta Odontol Scand. 2007; 65: 298-305. PubMed: https://www.ncbi.nlm.nih.gov/pubmed/18092202

20. Bartlett DW, Evans DF, Anggiansah A, Smith BG. The role of the esophagus in dental erosion. Oral Surg Oral Med Oral Pathol Oral Radiol Endod. 2000 Mar;89(3):312-315.

PubMed: https://www.ncbi.nlm.nih.gov/pubmed/10710455

21. Du X, Wang F, Hu Z, Wu J, Wang Z, Yan C, et al. The diagnostic value of pepsin detection in saliva for gastro-esophageal reflux disease: a preliminary study from China. BMC Gastroenterol. 2017; 17: 1-9. PubMed: https://www.ncbi.nlm.nih.gov/pubmed/29041918

22. Hayat JO, Gabieta-Somnez S, Yazaki E, Kang J-Y, Woodcock A, Dettmar P, et al. Pepsin in saliva for the diagnosis of gastrooesophageal reflux disease. Gut. 2015; 64: 373-380.

PubMed: https://www.ncbi.nlm.nih.gov/pubmed/24812000

23. Ocak E, Kubat G, Yorulmaz I. Immunoserologic Pepsin Detection in The Saliva as a Non-Invasive Rapid Diagnostic Test for Laryngopharyngeal Reflux. Balkan Med J. 2015; 32: 46-50.

PubMed: https://www.ncbi.nlm.nih.gov/pubmed/25759771

24. Spyridoulias A, Lillie S, Vyas A, Fowler SJ. Detecting laryngopharyngeal reflux in patients with upper airways symptoms: Symptoms, signs or salivary pepsin? Respir Med. 2015; 109: 963-969.

PubMed: https://www.ncbi.nlm.nih.gov/pubmed/26044812

25. Johnston N, Dettmar PW, Ondrey FG, Nanchal R, Lee SH, et al. Pepsin: biomarker, mediator, and therapeutic target for reflux and aspiration. Annals of the New York Academy of Sciences. 2018; 1433: 282-289.

PubMed: https://www.ncbi.nlm.nih.gov/pubmed/29774546

26. Wang YF, Yang CQ, Chen YX, Cao AP, Yu XF, et al. Validation in China of a non-invasive salivary pepsin biomarker containing two unique human pepsin monoclonal antibodies to diagnose gastroesophageal reflux disease. J Dig Dis. 2019; 20: 278-287.

PubMed: https://www.ncbi.nlm.nih.gov/pmc/articles/PMC6851552/

27. Dettmar P, Watson M, McGlashan J, Tatla T, Nicholaides A, Bottomley $\mathrm{K}$, et al. A Multicentre Study in UK Voice Clinics Evaluating the Noninvasive Reflux Diagnostic Peptest in LPR Patients. SN Comprehensive Clinical Medicine. 2019; 2: 57-65.

28. National Center for Biotechnology Information. Hydrochloric acid, $C I D=313$.

PubMed: https://pubchem.ncbi.nlm.nih.gov/compound/Hydrochloric-acid 